\title{
LA CONFUSIÓN DE LÍQUIDAS IMPLOSIVAS EN CASTILLA-LA MANCHA. UN ENFOQUE DESDE LA TEORÍA DE LA OPTIMIDAD*
}

\author{
Violeta Martínez Paricio \\ Centro de Ciencias Humanas y Sociales - CSIC \\ violeta.martinez@cchs.csic.es
}

\begin{abstract}
Resumen
Este artículo presenta un análisis fonológico dentro del marco de la Teoría de la Optimidad (Prince y Smolensky 1993) de la confusión de $r$ y $l$ implosivas en las hablas confundidoras de Castilla-La Mancha (Atlas Lingüistico y etnográfico de Castilla-La Mancha). Nuestra propuesta trata de explicar las causas que rigen la aparición de una u otra solución -lateral (beber $>$ bebel) y rótica (multa $>$ murta) - del fenómeno confundidor en la zona. Para ello, nos hemos basado en la importancia que adquieren en estas hablas las restricciones fonológicas universales relativas a las distancias de sonicidad (a partir de Clements 1990), así como las referentes a las exigencias de similitud entre segmentos adyacentes (Lombardi 1999; Colina 2007). Este análisis permite también dar cuenta de la confusión de líquidas en otras variedades (como el catalán hablado en Alguer, Cerdeña, o el castellano de algunos puntos del Caribe).

PALABRAS CLAVE: líquidas, posición implosiva, Teoría de la Optimidad, sonicidad.
\end{abstract}

\begin{abstract}
This paper puts forward a phonological analysis of the confusion of $r$ and $l$ in coda in some areas of Castilla-La Mancha (Atlas Lingüístico y etnográfico de Castilla-La Mancha) within the framework of Optimality Theory (Prince \& Smolensky 1993). Our claim tries to account for the factors which trigger the realisation of one or other output lateral (beber $>$ bebel) and rhotic (multa $>$ murta)- of the phenomenon. In order to achieve this goal, we have relied upon the prevalence in these varieties of the universal phonological constraints relative to the sonority distances (based on Clements 1990), and those requiring similarity between adjacent segments (Lombardi 1999; Colina 2007). The present analysis allows also accounting for the confusion of liquid consonants in other varieties, such as the Catalan spoken in Alguer (Sardinia) or the Spanish of some areas in the Caribbean.

KEYWORDS: liquid consonants, coda position, Optimality Theory, sonority
\end{abstract}

\section{Introducción}

A la luz de los datos del Atlas Lingüistico y etnográfico de Castilla-La Mancha' (ALeCMan), este artículo intenta describir el funcionamiento de la confusión de las líquidas implosivas (-r > - amol por amor, $-1>-\mathrm{r}$ farda por falda) en las hablas castellanomanchegas y ofrecer una explicación del fenómeno a partir de la metodología que proporciona la Teoría de la Optimidad (TO).

* Este trabajo se ha podido realizar gracias a la beca FPI (BES-2007-16190) concedida por el Ministerio de Ciencia e Innovación, adscrita al proyecto: Colaboración española al Atlas Linguistique Roman y al Atlas Linguarum Europae (HUM 2005-05372), desarrollado en el Instituto de la Lengua, la Literatura y la Antropología, del Centro de Ciencias Humanas y Sociales del CSIC, y a la ayuda de las codirectoras de mi trabajo de investigación Pilar García Mouton y Maria-Rosa Lloret Romañach.

1 Obra dirigida por García Mouton y Moreno Fernández. Gran parte del atlas — principalmente, la parte que se corresponde con la parte de la información léxica y la morfológica - se puede consultar en la red: http://www. 
La confusión de $r$ y $l$ en coda silábica es una característica que, a pesar de estar estigmatizada socialmente, se encuentra presente en variedades del español europeo (en puntos de Andalucía, de Extremadura, de Canarias, etc.) y del americano (en puntos de Cuba, de Puerto Rico, etc.). Un análisis fonológico de la confusión tendrá que tener en cuenta este hecho, así como la multiplicidad de soluciones dialectales de la misma. Por ello, hemos optado por un marco teórico como el de la optimidad, ya que, como se verá más adelante, estudios recientes han demostrado la idoneidad del modelo para dar cuenta de la variación lingüistica inherente a las lenguas.

A pesar de que existen numerosos estudios dialectales y de geografía lingüística sobre la confusión de líquidas en el español europeo ${ }^{2}$, estos no suelen ahondar demasiado en las causas específicas que llevan a los hablantes a confundir $r$ y $l$. La mayor parte explica la confusión desde un punto de vista articulatorio, aludiendo a cuestiones relacionadas con la minimización / maximización del esfuerzo articulatorio y a aspectos relacionados con la debilidad de la posición implosiva; sin embargo, no precisan, por ejemplo, por qué la confusión origina unas veces soluciones róticas y otras, laterales. Algunos de estos trabajos (Alarcos 1965; Quilis Sanz 1998) defienden la existencia de una tendencia generalizada a minimizar el esfuerzo en la producción del sonido, lo que, unido a la gran similitud articulatoria entre la $r$ y la $l$-en español, ambas son sonoras, se articulan en la región alveolar y con la lengua formando un resonador bucal amplio- propiciaría el "borrado" de las diferencias entre ambas, en situaciones de relajación articulatoria: en unos casos el relajamiento "suprimiría" el rasgo continuo de la $l$, que pasaría a realizarse como una rótica $(1>\mathrm{r}) \mathrm{y}$, en otros casos, se elidiría el rasgo interrupto de la $r$, realizándose entonces como una lateral $(r>1)$. Dentro de esta línea explicativa de la confusión de líquidas basada en factores articulatorios, hay trabajos que hacen más hincapié en la debilidad de la coda silábica para explicar su frecuente confusión (Alonso 1954; Malmberg 1965; Grammont 1971; García Jurado, Guirao y Rosso 1991); estos autores proponen que algunas características fonemáticas pertinentes en la tensión silábica, dejan de serlo en la distensión: esta posición experimenta una disminución de la fuerza articulatoria, lo que propicia la elisión de ciertas oposiciones en coda silábica, como la que diferencia entre $r$ y $l$, que apenas se oponen en sus rasgos. Aunque todas estas explicaciones resaltan aspectos interesantes de la confusión, no establecen las causas específicas por las que en unas variedades emerge la $r$, en otras la $l o$, incluso, existen variedades con ambas soluciones. Para que los argumentos anteriores fueran más adecuados deberían precisar cuál de las dos líquidas es más sencilla desde un punto de visto articulatorio - la menos costosa en su realización-y desde un punto de vista perceptivo - cual de las dos posee más pistas acústicas para su identificación-, lo que requeriría estudios de base fonética. Sin embargo, tales estudios no existen todavía. Además, también deberían matizar por qué en unas ocasiones se borran unos rasgos y no otros y qué es lo que propicia ese "borrado" de rasgos que afecta a las líquidas.

uah.es/otrosweb/alecman/. Para acceder al resto de información, se debe acudir directamente a los cuadernillos que contienen las respuestas a las encuestas dialectales, materiales que se encuentran inéditos en el CSIC y en la Universidad de Alcalá.

2 Alonso y Lida, 1945; Llorente, 1962 y 1965; Navarro Tomás, 1975; Catalán, 1989; Quilis-Sanz, 1998; González Salgado, 2003. 
Por todos estos motivos, a pesar de ser conscientes de las limitaciones de la TO, nos hemos servido de sus herramientas de trabajo para intentar dar cuenta de unos datos de campo que, anteriormente, no habían sido tratados. Estos datos resultan especialmente interesantes porque, a diferencia de otras variedades confundidoras que presentan una única solución a la confusión - lateral o rótica-, las hablas castellanomanchegas presentan ambas soluciones: en unos casos la rótica se convierte en lateral y, en otros, es la lateral la que se realiza como rótica. Es decir, este trabajo presenta un análisis fonológico que ha intentado dar cuenta de las diferentes soluciones de la confusión, así como de las causas que la desencadenan.

La estructura del artículo es la siguiente: en primer lugar, el apartado (2) describe la confusión de las líquidas en Castilla-La Mancha. A continuación, el apartado (3) introduce al lector en el marco teórico de la Optimidad, centrándose en los aspectos más relevantes para nuestro análisis, que se expone en (4) y se apoya, principalmente, en el trabajo de Clements (1990). Este apartado se divide en dos: en (4.1) se propone un análisis de la confusión en Castilla-La Mancha y, para probar su adecuación explicativa, en (4.2) se aplica a otras variedades confundidoras (al español de algunos puntos de América y al catalán hablado en Alguer, el alguerés). Finalmente, el apartado (5) recoge las conclusiones más significativas a las que se ha llegado y señala los principales problemas de nuestra propuesta, que tendrán que tratar de resolverse en futuras investigaciones.

\section{La confusión de $r$ y l en Castilla-La Mancha}

\subsection{Los atlas como fuente de datos lingüísticos}

Algunos estudios dialectales (Alonso y Lida 1945; Navarro Tomás 1975) habían constatado la presencia de la confusión de líquidas en algunas zonas de Castilla-La Mancha, sin embargo, la obra que permite describir con mayor precisión el comportamiento de la $r$ y la $l$ implosivas en las hablas castellanomanchegas es el Atlas Lingüistico y etnográfico de Castilla-La Mancha (ALeCMan). A finales de los ochenta y principios de los noventa, empezaron a completarse en 160 puntos de la comunidad las encuestas dialectales del ALeCMan. Los encuestadores se sirvieron en cada localidad de dos informantes ${ }^{3}$, uno representativo de cada sexo, de edad avanzada y, generalmente, procedentes del ámbito rural, lo que no habrá que perder de vista a la hora de interpretar nuestros datos ${ }^{4}$. La gran cantidad de puntos encuestados para el atlas lo convierten en un testimonio legítimo de la realidad lingüística de Casti1la-La Mancha. Por ello, para el análisis de la confusión de $r$ y $l$ en la zona, hemos elaborado una base de datos a partir de las palabras del atlas que contenían líquidas implosivas ${ }^{5}$ y las hemos organizado en distintos grupos, atendiendo a tres criterios, que pensamos podrían determinar la realización de las líquidas. Estos criterios fueron los siguientes: i) la situación

3 Exceptuando las ciudades, donde debido a su riqueza para estudios sociolingüísticos, se emplearon más informantes.

4 La confusión en estos hablantes de menor nivel de instrucción será siempre más frecuente que en hablantes más jóvenes o de niveles de educación más altos, quienes al sentir la confusión de líquidas como un rasgo impropio de la lengua correcta, tienden a corregirlo.

5 Para ello hemos empleado la cartografia de palabras disponible en red, así como las respuestas de los cuestionarios. 
relativa de la sílaba con $r$ o $l$ implosivas: en interior o final de palabra ii) la naturaleza del segmento que sigue a la líquida, en los casos en que la líquida está en interior de palabra ${ }^{6} \mathrm{y}$ iii) la posición del acento con respecto a la líquida. Una vez agrupadas las palabras en estos tres grupos, calculamos los porcentajes de confusión de cada contexto.

\subsection{Descripción de la confusión de líquidas en Castilla-La Mancha}

Gracias al trabajo de García Mouton y Moreno Fernández (1994) y a nuestra propia base de datos con los porcentajes de las respuestas del atlas, podemos afirmar que la confusión de $r$ y $l$ implosivas es un fenómeno muy variable y asistemático en Castilla-La Mancha. Con todo, existen tres focos donde la confusión se da con mayor intensidad. Estos núcleos confundidores se localizan en las provincias de Albacete, Ciudad Real y Toledo y se resaltan sombreados en la imagen 1:

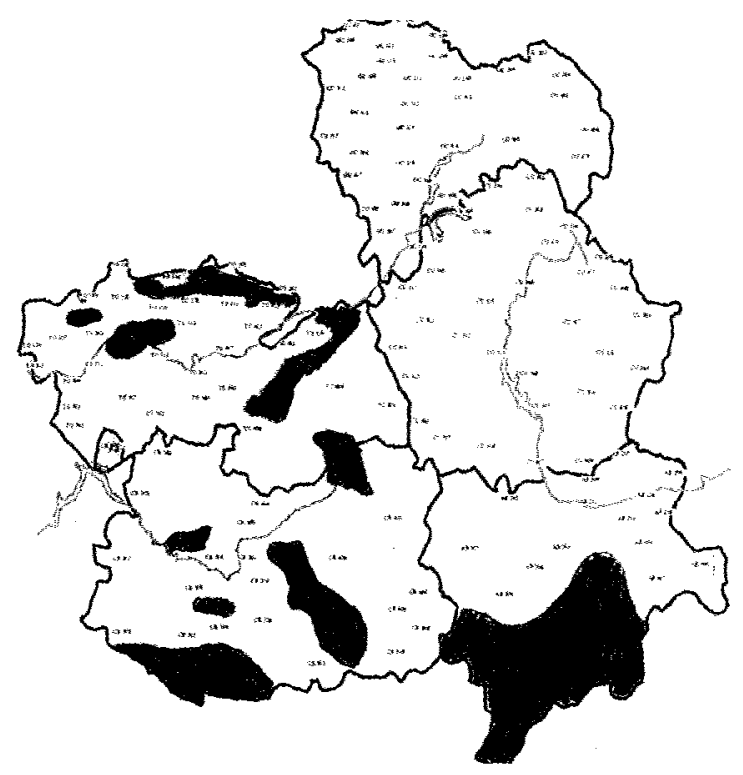

Imagen I: Principales focos confundidores en Castilla-La Mancha.

Esto no implica que en el resto de la comunidad no haya casos de confusión de líquidas?; sin embargo, las zonas sombreadas son las que confunden con mayor frecuencia, siendo Albacete la provincia con los porcentajes de confusión más elevados.

6 Cabe recordar que las respuestas a los cuestionarios sólo recogen respuestas aisladas en la cadena hablada y, además, en una situación de cierta formalidad. Por ello, en este trabajo sólo hemos tenido en cuenta el segmento que sigue a la liquida cuando ésta iba en interior de palabra; por tanto, para completar este estudio sería conveniente comparar nuestros datos con otros procedentes de conversaciones coloquiales y en los que, además, se analicen las realizaciones de las líquidas en la cadena hablada.

7 Por ejemplo, en el sur de Guadalajara, el informante masculino de Albalate de Zorita presenta esporádicamente confusión de líquidas. 
Tras revisar detenidamente los casos de confusión en cada grupo de palabras, hemos constatado que los tres focos presentan un comportamiento similar en lo que respecta a la confusión y que, además, ese comportamiento no responde a una asistematicidad total, sino que parece revelar la actuación conjunta de dos tendencias, formalizadas en (a) y (b):

a) Toda líquida implosiva a final de palabra en la forma subyacente tiende a realizarse como lateral. En otras palabras, toda rótica a final de palabra se transforma en lateral:

$$
\begin{aligned}
& \text { mar }>\text { mal } \\
& \text { comer }>\text { comel } \\
& \text { ajuar }>\text { ajual }
\end{aligned}
$$

b) Toda líquida implosiva en interior de palabra y seguida de oclusiva en la forma subyacente, tiende a realizarse como rótica. Es decir, una -1 implosiva seguida de oclusiva, suele convertirse en rótica:

$$
\begin{aligned}
& \text { multa }>\text { murta } \\
& \text { balcón }>\text { barcón } \\
& \text { talco }>\text { tarco }
\end{aligned}
$$

En conclusión, a partir de los materiales del ALeCMan, hemos comprobado que la confusión de líquidas presenta en Castilla-La Mancha dos soluciones: unas veces se confunde en favor de la lateral (a final de palabra) y otras, en cambio, se hace en beneficio de la rótica (en interior de palabra, seguida de oclusiva). Por lo tanto, un análisis satisfactorio del fenómeno tendrá que justificar esta doble solución, estas dos tendencias.

En (4) se expone una propuesta de análisis en términos de optimidad, pero antes de presentarla, veamos brevemente cómo funciona la teoría, cuáles son sus fundamentos y los motivos por los que pensamos que puede ser válida para dar cuenta de un fenómeno con tan variadas soluciones dialectales.

\section{La Teoría de la Optimidad}

\subsection{Concepción y funcionamiento de la gramática en la TO}

La TO nace en la década de los noventa con el trabajo de Prince y Smolensky (1993) y, en un primer momento, fue concebida para dar cuenta del funcionamiento del componente fonológico; sin embargo, con el tiempo se ha ido aplicando también a otros campos de la gramática $^{8}$. La TO posee una especial concepción de la gramática: ésta es el resultado de la ordenación específica del conjunto de restricciones lingüísticas universales. Dichas restricciones, presentes en la gramática universal (GU) de todo hablante, hacen referencia a las formas lingüísticas posibles en las lenguas y a la relación existente entre inputs y outputs lingüísticos relacionados. En definitiva, las restricciones no son más que formalizaciones de las tendencias observadas en los sistemas fonológicos de las lenguas (Parker 2002: 12) y, dependiendo de la jerarquía que adopten en las lenguas particulares, propician la emergencia de unas formas fonológicas $u$ otras, que siempre serán óptimas conforme a esa jerarquía. De ahí que la TO sea una teoría adecuada para dar cuenta de la variación lingüística: cada lengua o variedad ordena de una manera determinada las restricciones lingüísticas y esa je-

8 Para la confección de este apartado y el siguiente nos hemos basado en la introducción al modelo TO presentado por Jiménez y Lloret (2004). 
rarquía particular es la que justifica la variación. Se trata, por tanto, de una teoría lingüística que focaliza su atención, en las formas lingüisticas en si $^{9}$ e intenta determinar qué forma u output - de entre todos los posibles generados por una gramática-- es el mejor candidato, el candidato óptimo, para un input concreto, y los motivos que lo explican. Ello hace que la TO pueda resultar de gran utilidad a la hora de dar cuenta de un fenómeno como el de la confusión de $r$ y $l$, ya que en estos casos estamos ante un conjunto de inputs con $r$ y $l$ implosivas (amor, talco), y una serie de outputs que, unas veces, son fieles al input y mantienen todas las propiedades de la líquida $y$, otras, en cambio, la transforman en la otra líquida ( $r$ $>1$ : amol; $1>\mathrm{r}: \operatorname{tarco}$ ). Nuestro análisis tendrá que determinar las causas específicas -las restricciones- que propician la confusión de líquidas, las causas por las que algunos outputs, aun confundiendo las líquidas, resultan óptimos para determinadas hablas.

Una vez establecida la jerarquía de las restricciones de las variedades confundidoras de Castilla-La Mancha, ésta indicará cuál es la forma lingüistica o candidato óptimo, de entre todos los outputs posibles. Para ello, la gramática se vale de dos funciones universales: Generador (GEN) y Evaluador (EVAL). GEN, como su nombre indica, es la función encargada de generar el conjunto de candidatos posibles para un input determinado. Y Eval "evalúa" estos candidatos, estos outputs, basándose en una jerarquía de restricciones; es decir, Eval establece cuál es el candidato óptimo. Esta evaluación es global (Eval aplica la jerarquía de restricciones a todos los inputs) y paralela ${ }^{10}$ (sin estadios o niveles diferentes). El esquema de abajo, de Jiménez y Lloret (2004:115), ilustra cómo funciona la gramática, según la concepción de la TO:

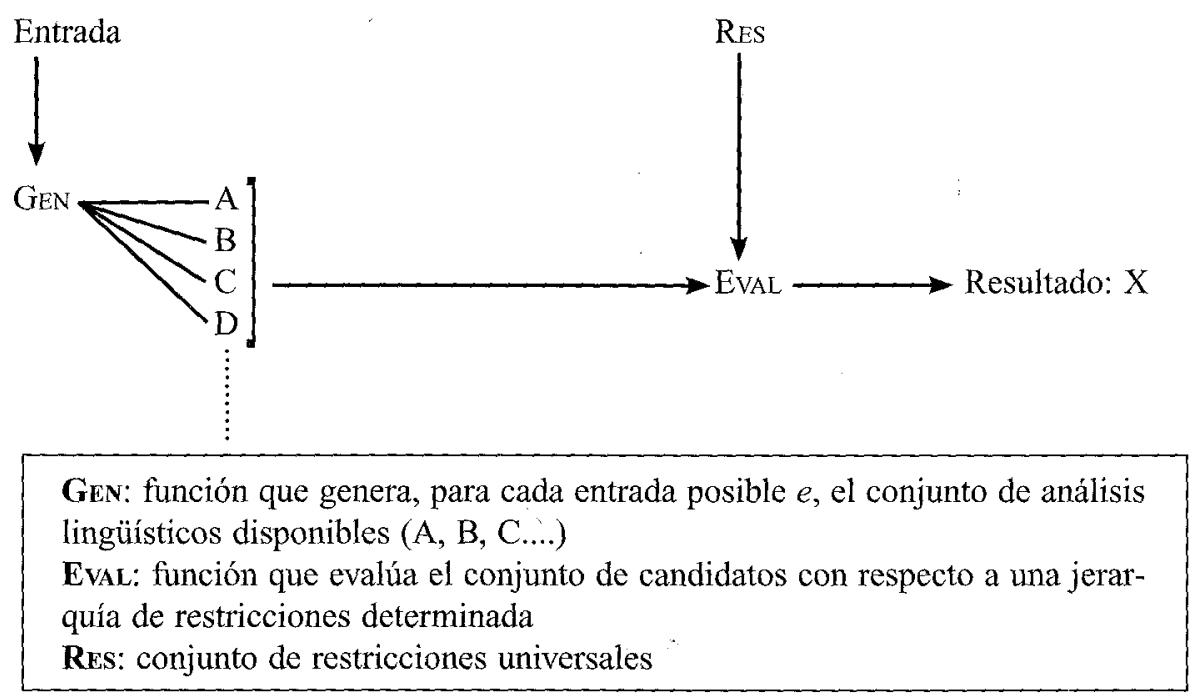

9 Frente a esto, los modelos fonológicos anteriores (las reglas de transformación fonológica del Sound Pattern of English, de Chomsky y Halle (1968) y los modelos generativos representacionales) focalizaban su atención en los procesos y transformaciones que llevaban de una forma (input) a otra forma (output).

10 Cabe señalar que también ha habido propuestas que admiten la existencia de distintos niveles en la evaluación; generalmente, tales propuestas han surgido cuando la TO ha tenido que dar cuenta de procesos vinculados con las relaciones intraparadigmáticas. La propuesta más simple, sin embargo, es la paralela. 


\subsection{Las restricciones lingüísticas}

Las restricciones lingüísticas son el fundamento de la teoría. A continuación se presentan las características de las más relevantes para nuestro análisis de la confusión.

Existen dos grandes familias de restricciones universales: las de FrdelidAD y las de MARCADEZ; cuando ambas entran en conflicto, la gramática es la encargada de resolverlo mediante el establecimiento de una ordenación específica de dichas restricciones. Esta universalidad de las restricciones no implica que tengan que ser respetadas por todas las lenguas; muy al contrario, las restricciones pueden violarse ${ }^{\mathrm{ll}}$. Cada lengua "decide" qué restricciones son más importantes en su jerarquía, qué restricciones respeta y cuáles viola, a pesar de que todas están presentes en la GU de los hablantes.

Las restricciones de fidelidad (FIDELIDAD) se basan en la relación existente entre el input y el output lingüístico; son las restricciones que "velan" por que haya la menor distancia posible entre ambas formas. Es decir, FIDELIDAD evalúa el grado de identidad entre inputs y outputs y penaliza las divergencias que puedan existir entre éstas. Las variedades que confunden las líquidas - que con un input como amor, pronuncian amol, por ejemploviolan las restricciones de fidelidad referentes a las propiedades de las líquidas. Algunas de las restricciones de fidelidad más relevantes para el estudio de la confusión son las de MAXIMIDAD (MAX), que penalizan los casos en que el output presenta, con respecto al input, elisión de material fónico (por ejemplo, una variedad que, ante un input como calor, tenga un output como caló estará violando MAX) y las de IDENTIDAD (ID), que postulan que los segmentos en correspondencia input-output (I-O) deben ser idénticos. Las restricciones de fidelidad pueden referirse a un segmento entero o, únicamente, a algunos de los elementos que lo componen. De este modo, hay familias de restricciones formadas por una restricción general y otras más específicas ligadas a ella. Así, la restricción general ID incluye otras restricciones específicas, como por ejemplo:

ID $_{\text {ataque }}$ : Los segmentos en el ataque en correspondencia I-O deben tener las misma propiedades.

ID $_{\mid+ \text {son| }}$ : Los segmentos en correspondencia I-O deben tener el mismo valor para el rasgo de sonoridad.

En nuestro análisis va a jugar un papel muy importante la restricción $\operatorname{ID}_{\text {ataque }}$ ya que en las hablas castellanomanchegas la confusión afecta a la posición implosiva, pero no a la explosiva, por lo que la restricción que obliga a conservar intactas las características del ataque ocupará un lugar dominante en su jerarquía. En lo que respecta a la relación entre las restricciones más específicas $\left(\operatorname{Res}_{\text {especifica }}\right)$ y las restricciones generales $\left(\operatorname{Res}_{\text {general }}\right)$, las específicas siempre se encontrarán en un lugar más importante de la jerarquía, dominando a la restricción general. Este hecho resulta evidente ya que:

Dado que Res general $_{\text {incluye todos los casos en que Res }}$ especifica $_{\text {es pertinente, la ordenación }}$ $\operatorname{Res}_{\text {especifica }} \gg \operatorname{Res}_{\text {general }}$ representa la única posibilidad para que Res específica $_{\text {tenga efectos }}$

11 Ello supone un avance frente a las teorías anteriores, ya que permite explicar muchos más fenómenos sin tener que recurrir constantemente a afirmaciones ad hoc para dar cuenta de las excepciones a los principios universales. 
visibles; de lo contrario, sus esfectos serían asumidos por $\operatorname{Res}_{\text {general }} y$, al no encontrar evi-

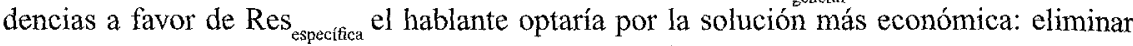
esta restricción de la gramática. (Jiménez, 1999:29) ${ }^{12}$

El conjunto de restricciones universales no está formado únicamente por las que aseguran la semejanza entre unas formas de entrada y otras de salida. Si esto fuera así, sería imposible dar cuenta de muchísimas formas lingüísticas que, por diversos motivos, desvían su forma superficial de la del input. Esto es precisamente lo que ocurre en los casos de confusión de $r$ y $l$, y se explica porque otro tipo de restricciones, las de marcadez (MARCADEZ), ocupan un lugar importante en la jerarquía de las variedades confundidoras. Las restricciones de marcadez centran su atención en la evaluación de outputs, seleccionando siempre los "menos marcados", los más sencillos; sin embargo, no siempre es fácil determinar qué formas o estructuras son las menos marcadas. Y es que la noción de marcadez es amplia y difusa, y todavía hoy no hay unos parámetros establecidos para determinarla. Se trata de un concepto relativo que tiene en cuenta diversos factores: cuantitativos, articulatorios y perceptivos. Para determinar si una estructura es marcada, por un lado, podemos observar si es o no es frecuente en las lenguas del mundo: cuanto mayor sea su presencia en las lenguas, menos marcada será la estructura, ya que el hecho de que sea más simple le permite "emerger" con mayor frecuencia en las diferentes lenguas. Por otro lado, para determinar la marcadez de una estructura también se tienen en cuenta factores articulatorios y perceptivos. Así, las estructuras "más simples" son las que articulatoriamente requieren un menor esfuerzo gestual $\mathrm{y}$, desde un punto de vista perceptivo, presentan más contrastes para su identificación, presentan mayor solidez en sus pistas auditivas ${ }^{13}$. De hecho, en los últimos años, se ha venido desarrollando una teoría fonológica en esta línea, con fuerte base fonética, que tiene entre sus objetivos explicar los procesos fonológicos y la motivación de las restricciones partiendo de la percepción. Para esta teoría, que contribuirá sin duda al desarrollo de la TO, las estructuras menos marcadas son las "más perceptibles". Por ejemplo, para el análisis de la confusión de líquidas en coda silábica es muy interesante constatar que, desde el punto de vista de la percepción, la posición implosiva es más débil que la explosiva. Como señala Wright (2004), numerosos tests perceptivos evidencian que:

Onset transitions are less vulnerable to environmental masking than offset transitions. This is not to say that coda transitions necessary contain less information, but rather that in any but the best listening environments they will stand a poorer chance of reliably transmitting information about a particular contrast. (p. 46)

Esto explicaría por qué la $r$ y la $l$ se confunden en mayor medida en posición implosiva, posición en la que es más difícil percibir ciertos contrastes.

Para ejemplificar el concepto de marcadez, veamos cuál es la sílaba menos marcada, la sílaba óptima universal atendiendo a su marcadez. Esta resulta ser la que posee un ataque consonántico, un núcleo vocálico, y no posee coda: [CV]. Desde un punto de vista articulatorio, esta estructura maximiza la distancia entre las consonantes y las vocales, lo que

12 La traducción al castellano es nuestra, puesto que el original está en catalán.

13 Una pista auditiva es la información en la señal acústica que permite al oyente captar la existencia de contrastes fonológicos (Wright, 2004). 
permite, perceptivamente que las dos clases (consonantes y vocales) queden bien definidas (Jiménez 1999: 48, basándose en el trabajo original de Clements 1990). Además, todas las lenguas admiten estas sílabas, lo que evidencia también su falta de marcadez.

Como avanzábamos, las restricciones lingüísticas constituyen formalizaciones de las tendencias universales observadas en las lenguas. Por ello, muchas se formulan a partir de las escalas lingüísticas universales. Así, la escala universal de sonicidad va a desempeñar un papel fundamental a la hora de establecer las restricciones que pueden dar cuenta del fenómeno confundidor. El grado de sonicidad de los segmentos se basa, articulatoriamente, en el grado de abertura del tracto vocal y, perceptivamente, en la mayor perceptibilidad de los segmentos con mayor grado de sonicidad (Bonet y Lloret, 1998: 65). En la actualidad, la mayor parte de estudios (Côté 2000; Wright 2004; Lloret y Jiménez 2007) coinciden en su propuesta de la escala universal de sonicidad:

$$
\begin{aligned}
& \text { (1) Vocales }>\text { Glides }>\text { Líqudas }>\text { NaSales }>\text { Fricativas }>\text { OCLUSivas, [f] } \\
& \text { Mayor sonicidad }
\end{aligned}
$$

Aunque podría pensarse que la [f] debería estar situada junto al resto de fricativas (más abiertas y, por tanto, más sonantes que las oclusivas), su especial comportamiento -es el único segmento fricativo que, al igual que las oclusivas, puede formar ataques complejos (flan, fresa) - la sitúa en el mismo nivel que las oclusivas (más cerradas y, por tanto, menos sonantes). Por otro lado, observamos que esta escala general sitúa a las líquidas en un mismo nivel. Sin embargo, para nuestro análisis será conveniente fijar la escala de sonicidad relativa de las líquidas. La escala de sonicidad es esencial para la comprensión de diversos fenómenos fonológicos porque determina la configuración de la estructura silábica: los segmentos que forman una sílaba no aparecen en cualquier orden, sino que se organizan conforme a la escala de sonicidad (Clements 1990). Así, los segmentos con mayor grado de sonicidad se sitúan en el núcleo silábico y, a medida que nos alejamos de este, la sonicidad disminuye ${ }^{14}$ :

(2)

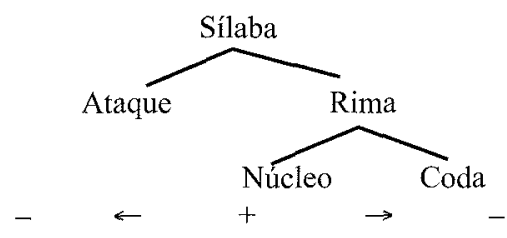

Todas estas afirmaciones entorno a la estructura silábica y al grado de sonicidad de los elementos que la componen serán tenidas en cuenta en (4) a la hora de establecer la jerarquía de restricciones de las variedades confundidoras en Castilla-La Mancha.

14 Esta estructura no se cumple absolutamente en todas las lenguas. Así, hay lenguas como el inglés con nasales y líquidas como núcleos silábicos, a pesar de que el grado de sonicidad de estas sonantes es más bajo que el que presentan las vocales $y$, por tanto, no constituyen los mejore núcleos. Con todo, esta estructura suele funcionar en las lenguas romances, por lo que nos hemos basado en ella para nuestro análisis. 


\section{Análisis de la confusión en términos de Teoría de la Optimidad}

\subsection{Análisis del fenómeno confundidor en Castilla-La Mancha}

El objetivo de este análisis es establecer la gramática de las hablas confundidoras en Castilla-La Mancha; determinar las restricciones que entran en conflicto en estas variedades y la manera específica en que lo resuelven. Para ello, recordemos esquemáticamente los entornos que tienden a presentar confusión en estas hablas ${ }^{15}$ :
a) - Vocal $[r]$ \#
$>\quad$ Vocal [I] \#
$($ comer $>$ comel $)$
b) - [1].oclusiva
$>\quad-[$ [ ].oclusiva
(rulta $>$ murta)

En primer lugar habrá que esclarecer qué peculiaridades tienen (a) y (b) para desencadenar los cambios $l>r$ y $r>l$; en segundo lugar, tendremos que intentar aclarar qué restricciones de marcadez se sitúan, en estas variedades, por encima de las de fidelidad relativas a las propiedades de las líquidas y a la posición implosiva, ocasionando de este modo la confusión. Para ello, veamos previamente el trabajo de Clements (1990), ya que proporciona claves importantes para nuestro análisis, especialmente a la hora de establecer las diferentes restricciones de marcadez relevantes en las gramáticas de las variedades confundidoras. Se trata de un estudio que centra su interés en la escala universal de sonicidad y en la estructura silábica; en él también se han basado otros trabajos enmarcados en la TO (Martínez-Gil 1997; Bat-El 1996) para dar cuenta de diversos cambios lingüisticos. Estos trabajos, si bien no han analizado específicamente la confusión, proponen que ciertos cambios en las sílabas se dan, precisamente, para mejorar las distancias de sonicidad entre los segmentos que las componen. Son estudios que postulan restricciones de marcadez relativas a las distancias de sonicidad intrasilábicas (entre los segmentos de una misma silaba) y a las distancias de sonicidad intersilábicas (entre el último segmento de una silaba y el primero de la sílaba adyacente). ¿Pero a qué hacen referencia exactamente estas distancias de sonicidad? ¿Y por qué son relevantes para el estudio de la confusión? Para responder a estas preguntas, vayamos primero al trabajo de Clements (1990).

Clements (1990) atribuye un valor específico a cada segmento de la escala de sonicidad y, a partir de Murray y Venneman (1983), formula una serie de principios entorno a la distribución de la sonicidad en la sílaba, lo que le permite determinar cuáles son los mejores contactos intra- e intersilábicos ${ }^{16}$. En este trabajo es fundamental la noción de semisílaba: cada sílaba consta de una semisílaba inicial y de otra final. Por ejemplo, una sílaba como [kran] consta de las semisilabas [kra, an] y la sílaba [pa], de [pa, a]. La idea subyacente al concepto de semisílaba es que el perfil de sonicidad de la primera parte de la sílaba es independiente al perfil de sonicidad de la segunda parte. Así, Clements defiende que la sílaba óptima es la que maximiza la distancia en sonicidad de los elementos de su semisílaba

15 La aimohadilla (\#) señala el final de palabra y el punto (.) el límite silábico.

16 Clements no emplea la escala presentada en (1) para el cálculo de las distancias intra- e intersilábicas, sino que utiliza una más general: Vocales $>$ Glides $>$ Liquidas $>$ Nasales $>$ Obstruyentes. 
inicial, pero minimiza la sonicidad de sus elementos en la semisílaba final. Según esto, la mejor semisílaba inicial sería una del tipo [OclusivaVocal], ya que las oclusivas y las vocales, como se desprende de la escala propuesta en (1), son los segmentos cuyo perfil de sonicidad más alejado se encuentra; por su parte, la mejor semisílaba final sería la que, al contrario, minimiza esa distancia de sonicidad, es decir [VocalGlide]. El autor va más allá y propone también cuáles son los mejores y los peores contactos intersilábicos: cuanta más distancia haya entre el último segmento de una sílaba y el primero de la sílaba adyacente, más cerca estaremos del contacto intersilábico óptimo, tanto por cuestiones articulatorias como perceptivas. Además, el segmento final de una sílaba debe presentar un mayor grado de sonicidad que el primer segmento de la sílaba siguiente. Así, un contacto del tipo [Vocal. Oclusiva] como el de [pa.ta] constituiría un contacto óptimo. Por último, en lo referente a las sílabas con ataques complejos, Clements señala que en estos casos, la sílaba que tenga la sonicidad distribuida de la manera más homogénea posible, será la óptima (p. 304).

Ahora ya sí, una vez revisadas estas generalizaciones sobre la sílaba, retomemos los casos que presentan confusión en Castilla-La Mancha. En primer lugar, si reflexionamos entorno al contexto (a) A final de palabra, las rótica percusivas se transforman en lateral (-Vocal [ $\mathrm{C}]$ \# > Vocal [1] \#) e intentamos determinar qué resulta mejor, atendiendo a la distancia intrasilábica, si una lateral [VocalLateral] o una rótica [VocalRótica], constatamos que la líquida que más cerca en sonicidad esté de las vocales, será la que forme la mejor sílaba, por minimizar la distancia de sonicidad entre los elementos de la semisílaba final. Con todo, ya vimos que la escala universal de sonicidad (1) no diferenciaba entre las líquidas, sino que las dotaba de un mismo valor de sonicidad. Por ello, es de especial relevancia el trabajo teórico y empírico de Parker (2002) que propone una ordenación específica de las líquidas atendiendo a su grado de sonicidad. Parker (p. 233) presenta evidencias suficientes - especialmente contundentes en español- acerca de la legitimidad de la siguiente escala relativa a las líquidas:

(3) LATERAL [l] > Rótica PERCUSIVA [r] $>$ Rótica VIBRANTE [r]

[+sonicidad] [-sonicidad]

La nueva escala de sonicidad, con sus respectivos valores, será la siguiente:

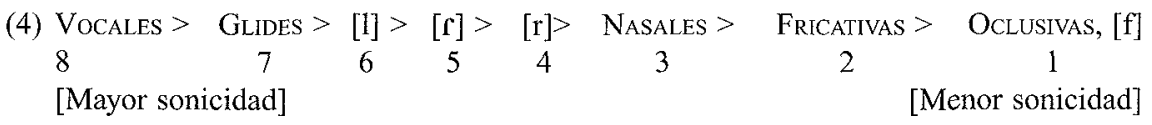

A partir de esta escala y teniendo en cuenta que existe una preferencia por la menor distancia intrasilábica entre la coda y el núcleo que la precede, estamos en condiciones de afirmar que las mejores sílabas con líquidas implosivas serán las que presenten la lateral, ya que entre la vocal (V) y la lateral (L) hay menor distancia de sonicidad que entre la vocal y la rótica percusiva $(\mathrm{R})$ :
(5) $\mathrm{VL}=8-6=2$
$\mathrm{VR}=8-5=3$
(-) Mejora la distancia intrao ${ }^{17}$ de la coda

17 Utilizaremos la abraviatura intrao para intrasilábica e intero para intesilábica y el símbolo o para la sílaba. El símbolo :) indica que una sílaba es mejor que otra, y $*$, peor. 
Todo lo cual evidencia el posible detonante del cambio $\mathrm{r}>\mathrm{I}$ a final de palabra, característico de las hablas confundidoras de Castilla-La Mancha: la $r$ se convertiría en $l$ para mejorar la distancia intrasilábica de la coda, ya que la -1 implosiva resulta mejor que la $-r$, por ser más sonante (la lateral) y estar más cerca su sonicidad de la de la vocal. En términos de optimidad esto se explicaría debido a que la restricción de marcadez encargada de favorecer las sílabas con las mejores distancias intrasilábicas en las codas ocupa un lugar muy importante en la jerarquía de las variedades confundidoras de Castilla-La Mancha. Esta restricción de marcadez es la siguiente:

SONICIDADINTRASILÁBICA voda $_{\text {: }}$

Cuanto menor sea la distancia de sonicidad entre el núcleo y su coda, mejor "formada" estará la sílaba. Abreviatura de la restricción: SONINTRAo ${ }_{\text {coda }}$

Y se situará por encima de otras restricciones de fidelidad, como las que obligan a mantener las propiedades de los segmentos del input intactos en el output, lo que impediría el cambio de $r>1$ y, en definitiva, cualquier tipo de confusión:

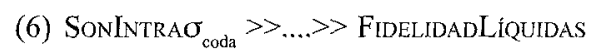

Retomando ahora el segundo entorno que tendía a presentar confusión, el contexto (b) En interior de palabra y seguida de oclusiva, una lateral implosiva se transforma en rótica (ol.oclusiva > oroclusiva), observamos que en este caso la confusión -el cambio de l> $\mathrm{r}$ - no se da para mejorar las distancias de sonicidad, ya que este cambio las empeora (tanto las intrasilábicas de la coda como las intersilábicas):

(7) Cambio de $1>\mathrm{r}$ en multa $>$ murta:

- Empeora la distancia intrasilábica, [VocalLíquida]: multa: $\mathrm{VL}=8-6=$ distancia intrao de 2

murta: VR $=8-5=$ distancia intrao de $3 \oplus$ Empeora la distancia y viola fidelidad

- Empeora la distancia intersilábica, [Líquida.Oclusiva]:

multa: L.Ocl, $=6-1=$ distancia intero de 5

murta : .Ocl. $=5-1=$ distancia inter $\sigma$ de $4 \odot$ Empeora la distancia y viola fidelidad

La restricción de marcadez que hace referencia a las distancias intersilábicas fue propuesta, entre otros, por Bat-El (1996: 305), a partir de Clements (1990: 287):

oCONTACTSLOPE: Cuanto mayor sea la distancia en sonicidad entre el ataque y el último segmento de la sílaba precedente, mejor.

Abreviatura de la restricción: oCONTSLOPE

Como se desprende de la definición de oContSlope, para afirmar que dos sílabas presentan una buena (o mala) distancia intersilábica no contamos con unos valores fijos establecidos como "óptimos" (por ejemplo "la distancia de sonicidad de 5 es la óptima"); al contrario, se trata de dar con la mejor combinación posible de sonidos, es decir, la mejor distancia intersilábica no es constante sino relativa. De ahí que se pueda establecer una 
gradación; por ejemplo, dentro de las consonantes sonantes, la que "mejor combina con una oclusiva" será la lateral:

$$
\begin{aligned}
& \text { (8) L. Oclusiva }=6-1=5 \odot \quad \text { (mejor distancia inter } \sigma \text { ) } \\
& \text { R. Oclusiva }=5-1=4 \\
& \text { N. Oclusiva }=4-1=3 \Leftrightarrow \quad \text { (peor distancia intero) }
\end{aligned}
$$

Volviendo a (7), donde se ilustra mediante un ejemplo el contexto (b), constatamos que en las hablas confundidoras de Castilla-La Mancha el cambio $1>r$ se da a pesar de que la rótica empeora las distancias de sonicidad. Este cambio, además, no solo viola las restricciones de marcadez referentes a las distancias de sonicidad ( $\sigma$ CONTSLOPE, SonInTRa $\sigma_{\text {coda }}$ ) sino que también viola las restricciones de fidelidad (ya que la lateral del input no se mantiene en el output). Por ello, dado que en este contexto la confusión de líquidas no está motivada ni por el intento de mejorar las distancias de sonicidad, ni tampoco por las exigencias de fidelidad entre inputs y outputs, tendrá que haber otra restricción de marcadez que desempeñe un papel decisivo en las hablas confundidoras de Castilla-La Mancha y será la responsable del cambio $(1>\mathbf{r})$ en interior de palabra. Para averiguar de qué restricción se trata, focalizaremos de nuevo la atención en el contexto (b) (ol.oclusiva > or.oclusiva) e intentaremos determinar qué tiene de especial para desencadenar este cambio "inesperado"; inesperado en el sentido de que el output no mejora las distancias de sonicidad (no respeta las restricciones de marcadez hasta ahora vistas), pero tampoco es fiel a las propiedades del input (viola FIDELIDAD).

Según nuestros datos, las líquidas se confunden en interior de palabra cuando les sigue una oclusiva, por lo que parece que este tipo de consonantes debe de tener alguna propiedad, algún rasgo, que propicie la transformación de la lateral en la rótica en tales contextos. Más concretamente, la clave del cambio $1>\mathrm{r}$ parece estar en alguna de las propiedades que comparten las oclusivas y la rótica percusiva, puesto que las oclusivas son los segmentos que desencadenan el rotacismo de la [1] en las variedades de Castilla-La Mancha. Como apuntábamos en la introducción, algunos trabajos (Alarcos 1965; Quilis-Sanz 1998) habían constatado que la gran semejanza entre la $r$ y la $l$ junto a la tendencia general a la minimización del esfuerzo articulatorio eran los causantes de su frecuente confusión. Los defensores de estas propuestas alegaban que la $r$ y la $l$ tan solo se oponían en uno de sus rasgos: mientras que las róticas eran intermitentes o interruptas, las laterales eran continuas. De modo que, si se suprimía uno de estos rasgos, la percusiva se convertía en lateral y viceversa. Aunque este argumento de la relajación articulatoria sólo era capaz de explicar un tipo de cambio, resulta de interés para nuestro análisis en TO, ya que propone una interesante descripción de las líquidas. Atendiendo a esta caracterización, defendida también por el resto de estructuralistas, consideraremos que las líquidas sólo se oponen por el rasgo interrupto (de la $r$ ) frente al continuo (de la $l$ ), ya que ambas son dorsales y líquidas:

$$
\begin{aligned}
& / \mathrm{r} /:[\text {-continua }] \\
& / \mathrm{L} / \mathrm{:}+\text { continua }]
\end{aligned}
$$

Si relacionamos ahora esta observación con las propiedades de las oclusivas, constatamos que las róticas y las oclusivas comparten el rasgo [-continuo]; es decir, el hecho de que la $r$ se encuentre más próxima a las oclusivas (por el hecho de poseer este mismo rasgo) podría motivar el cambio $1>\mathrm{r}$. En tales casos, la $l$ dejaría de ser continua al entrar en contac- 
to con una oclusiva y, de este modo, emergería la rótica. Además, sabemos que la posición de coda es una posición débil frente a otras posiciones fuertes, como el núcleo o el ataque; es decir, hay una serie de razones psicolingüísticas y fonéticas que convierten la coda en una posición menos perceptiva que el resto, lo que implica que los rasgos asociados a esta posición débil sean más difícil de mantener que los rasgos asociados a los segmentos fuertes (Jiménez y Lloret 2004). Por lo tanto, en TO, las restricciones que exigen la conservación de los rasgos en posiciones perceptivamente fuertes (como por ejemplo $\mathrm{ID}_{\text {ataque }}$ ) estarán por encima de las restricciones que exigen que se mantengan los rasgos de las posiciones débiles (por ejemplo, $\mathrm{ID}_{\text {coda }}$ ) (Beckman 1999). Todo lo cual permitiría explicar por qué la confusión de líquidas en las hablas castellanomanchegas (y en la mayoría de variedades confundidoras) afecta a la posición implosiva. Al ser ésta una posición débil, es normal que no pueda conservar siempre sus rasgos "intactos" y se vea influida por los segmentos adyacentes. Esto es lo que ocurriría, por ejemplo, cuando las nasales implosivas modifican su punto de articulación al entrar en contacto con otros segmentos adyacentes (por ejemplo, una nasal seguida de una velar, modifica su punto de articulación coronal y se realiza como velar) y, también, cuando la lateral implosiva se transforma en rótica percusiva seguida de oclusiva. En TO, la restricción que capta esta última transformación (L.Oclusiva $>$ R.Oclusiva) es la restricción de marcadez que relaciona los segmentos adyacentes y exige que estos compartan algún rasgo: CompartiR. Esta sería la restricción más general y ocuparía un lugar más bajo en la jerarquía con relación a la restricción específica que exige que se comparta el rasgo concreto [ $[ \pm$ continuo]:

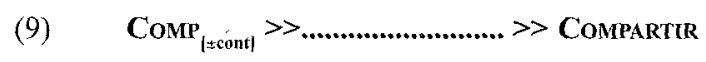

Estas restricciones fueron propuestas para poder explicar los procesos de asimilación en las lenguas. Así, Colina (2007), basándose en Lombardi (1999), proponía la existencia de una restricción específica de la familia COMParTIR $\left(\mathrm{COMP}_{[ \pm \text {sonoro }]}\right)$ para poder dar cuenta de la asimilación del rasgo sonoro de las oclusivas sordas implosivas, seguidas de segmentos sonoros. Este hecho le permitía explicar las frecuentes asimilaciones en sonoridad en castellano: situando $\operatorname{CoMP}_{[ \pm s o n]}$ dominando a $\mathrm{ID}_{[ \pm s o n]}$. Por ejemplo, le permitía explicar por qué algunos hablantes pronunciaban como $[\delta]$ la $[\mathrm{t}]$ de fítbol:

\begin{tabular}{|l|c|c|}
\hline /fútßol/ & CoMP $_{\text {ltsonl }}$ & ID $_{\text {[tson] }}$ \\
\hline b. fúd. Bol & & $*$ \\
\hline
\end{tabular}

Antes de seguir con nuestro análisis, cabe señalar que la caracterización de $r$ como [-continuo] y $l$ como [+continuo] es un tema problemático en la bibliografia, puesto que ha habido otras propuestas - especialmente, desde el marco de la fonología generativa- que las caracterizaron a la inversa: $r$ como [+continuo] y $l$ [-continuo]. Así, Chomsky y Halle (1968) afirman que las variantes de [r] son continuas. Sin embargo, estos autores se basan fundamentalmente en las $[r]$ inglesas, que son más vocálicas que las españolas. En cuanto a la [1], los autores constatan que su caracterización como [ \pm continuo] es "complicada": 
The characterization of the liquid [1] in terms of continuant-non continuant scale is even more complicated. If the defining characteristic of the stop is taken (as above) as total blockage of air flow, then [1] must be viewed as a continuant (...). If, on the other hand, the defining characteristic of stops is taken to be blockage of air flow past the primary stricture, then [1] must be included among the stops. (p. 318)

A pesar de que no hay acuerdo entorno a la caracterización de las líquidas atendiendo al rasgo [ \pm continuo], aquí nos acogeremos a la postura estructuralista, que siempre ha mantenido que la vibrante es la líquida interrupta o intermitente frente a la lateral, que es la líquida continua $^{18}$. Sin embargo, no hay que perder de vista que esta caracterización es, cuanto menos, problemática y, además, si tenemos en cuenta la propuesta de algunos trabajos sobre la espirantización de las oclusivas sonoras (Bonet y Lloret 1998; Palmada 1997), nuestra postura podría verse debilitada. Estos trabajos defienden que la espirantización de las oclusivas sonoras se da por la expansión del rasgo [+continuo] de los segmentos adyacentes; así, por ejemplo, la realización de alba sería [alßa], con espirantización. Si esto fuera totalmente así, nuestra propuesta, que señala que el valor [-continuo] de la oclusiva es el desencadenante del cambio l> r, no sería válida para el caso de las oclusivas sonoras, ya que -teniendo en cuenta los trabajos sobre la espirantización- dichos segmentos más que oclusivas sonoras (caracterizadas por el rasgo [-continuo]) serían espirantes (es decir, poseerían el rasgo [+continuo] y, por lo tanto, el cambio $1>\mathrm{r}$ no podría explicarse por la expansión del rasgo [-continuo]). Con todo, y aunque queda lejos de nuestro objetivo dar cuenta aquí del proceso de espirantización, cabe señalar que la explicación de la espirantización como expansión del rasgo [+continuo] no funciona para todos los casos (por ejemplo, la lateral no espirantiza la dental oclusiva sonora cuando la precede, soldado: [soldado]) y, además, también hay interpretaciones de la espirantización que achacan el fenómeno a otras causas y no a la intervención del rasgo [ \pm continuo ${ }^{19}$. Por todos estos motivos, pensamos que, de momento, puede seguir manteniéndose la caracterización de la $l$ como [+continua] y la $r$ [-continua]. En favor de nuestro análisis subrayamos que, al vincular los cambio de $1>r$ con el valor del rasgo [ \pm continuo], es capaz de predecir correctamente un resultado importante: el hecho de que los cambios $1>r$ ante oclusivas sordas (indudablemente [-continuas] $)$ son más numerosos que ante sonoras, como reflejan los porcentajes.

Si retomamos ahora nuestro análisis y aplicamos la nueva restricción de marcadez $\operatorname{Comp}_{[ \pm c o n t]}$ a los datos sobre la confusión en Castilla-La Mancha, obtenemos la jerarquía de restricciones universales propuesta en (10):

(10) Jerarquía de las hablas confundidoras de Castilla-La Mancha:

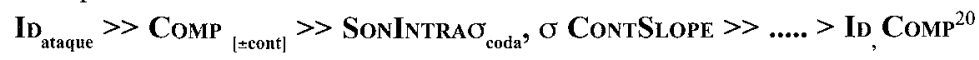

18 Para una caracterización alternativa de las consonantes sonantes en el marco de la fonología autosegmental, véase Lloret (1997).

19 Así, por un lado, Mascaró (1991) defiende el carácter meramente fonético (y no fonológico) de este fenómeno. Por otro lado, Piñeros (2001), basándose en el trabajo de Kirchner (1998), interpreta la espirantización del español en las codas como una estrategia para presentar consonantes sonoras en esta posición (en contraste, como en ved [béð] vs. vez [bé $\theta]$, o de tipo asimilatorio, como en fútbol, véase (9)). Asimismo, en esta línea, Wheeler (2005) apunta que en catalán -y es extensible al español- la espirantización en ataque silábico puede ser interpretada como un mero proceso de lenición (y no asimilatorio) en términos de la restricción LAZY ("Minimize articulatory effort", Kirchner, 1998: 38). 20 Las dos restricciones (ID y COMP) así como las referentes a las distancias de sonicidad no se encuentran ordenadas entre sí puesto que no hay evidencia de un orden específico para ellas; esto se marca mediante las comas. 
A continuación comprobaremos mediante varias tablas de optimidad que la jerarquía de restricciones de (10) selecciona, efectivamente, el candidato adecuado (no consideraremos en las tablas las restricciones ID y COMP, puesto que se encuentran en un lugar muy bajo de la jerarquía y, por lo tanto, no son relevantes en la selección del candidato óptimo). En la evaluación, el símbolo $\left({ }^{*}\right)$ señala el candidato óptimo, los asteriscos $\left(^{*}\right)$ indican que una forma viola una restricción, la exclamación (!) pone de manifiesto que la violación es fatal (es decir, dicha forma queda descartada inmediatamente en la evaluación) y las líneas discontinuas indican que dos restricciones no están ordenadas entre sí.

(11)

\begin{tabular}{|c|c|c|c|c|}
\hline /múlta/ & $I_{\text {ataque }}$ & $\operatorname{CoMP}_{[ \pm c o n t}$ & SonINTRA $\sigma_{\text {coda }}$ & oContSLop \\
\hline a. múl. ta & & $* !$ & & \\
\hline$\circledast$ b. múr. ta & & & * & $*$ \\
\hline
\end{tabular}

A pesar de que [múr.ta] viola más restricciones que [múl.ta], como en esta variedad la restricción que exige que dos segmentos adyacentes concuerden en el rasgo [ \pm cont] se encuentra en un lugar dominante en la jerarquía, el candidato (11b) resulta el óptimo. De este modo, queda comprobado como la jerarquía de (10) da cuenta de la tendencia (b) [ol.oclusiva] $>$ [or.oclusiva], propia de los puntos confundidores de Toledo, Ciudad Real y Albacete. Veamos ahora si esta jerarquía también da cuenta de la tendencia (a) $[-$ or \#] $>[-\infty 1 \#]$ :

\begin{tabular}{|c|c|c|c|c|c|}
\hline /aorác/ & $\mathrm{ID}_{\text {ataque }}$ & $\operatorname{CoMP}_{\{\neq \operatorname{cont}\}}$ & SONINTRAO $\sigma_{\text {coda }}$ & oCONTSLOPE & ID \\
\hline a. a.o.rár & & $*$ & $* !$ & & \\
\hline Fb. a.o.rál & & $*$ & & & * \\
\hline
\end{tabular}

La jerarquía de (10) también da cuenta del paso $r>1$ en las hablas castellanomanchegas: el candidato (12b) presenta una distribución de la sonicidad intrasilábica de la coda mejor que la del candidato (12a) y por ello, aunque (12a) sea más fiel al input, (12b) resulta el candidato óptimo. Ahora, tan solo queda comprobar que esta jerarquía no modifica los ata-. ques complejos formados por líquidas, ya que en las variedades castellanomanchegas estos grupos se mantienen intactos. Incluimos ahora la restricción relativa a la sonicidad de los ataques complejos, basada en Clements (1990), SonINTRa $\sigma_{\text {ataquecomplejo }}$ y la de MAXIMIDAD -elididas antes por no ser relevante-- y obtenemos la siguiente jerarquia:

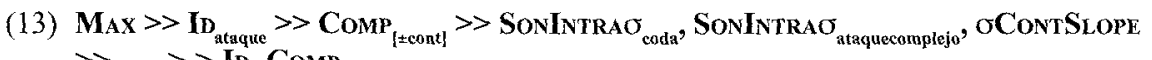
$>\ldots . .>>\mathrm{ID}, \mathrm{COMP}$ 
(14) Veamos si funciona mediante la evaluación de plaza:

\begin{tabular}{|c|c|c|c|c|c|c|}
\hline /pláea/ & MAX & $\mathbf{I D}_{\text {ataque }}$ & CoMP $_{\text {|tonant }}$ & SONINTRA $\sigma_{\text {coda }}$ & SoNINTRA $\sigma_{\text {ataqueconplecjo }}$ & бCONTSLOPE \\
\hline a. plá. $\theta a$ & & & $*$ & & * & \\
\hline b. prá. $\theta \mathrm{a}$ & & $* !$ & & & & \\
\hline c. pá. $\theta$ a & $* !$ & & & & & \\
\hline
\end{tabular}

El candidato [pá. $\theta$ a] queda inmediatamente descartado, ya que suprime material fónico del input (el segundo segmento del ataque complejo), es decir, viola MAX, que ocupa un lugar dominante en la jerarquía. Por su parte, aunque el ataque complejo del candidato

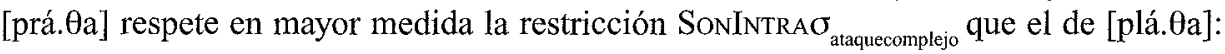

$$
\begin{array}{ll}
p l a & p r a \\
168 & 158 \quad \text { Sonicidad distribuida de manera más uniforme }
\end{array}
$$

también queda fuera de la evaluación por violar una restricción de fidelidad, la referente a las propiedades del ataque $\left(\mathrm{ID}_{\text {ataque }}\right)$. El candidato óptimo, por tanto, es el primero, lo que evidencia que la jerarquía propuesta parece funcionar en las variedades confundidoras castellanomanchegas.

El inconveniente principal de la ordenación de restricciones hasta ahora propuesta es que, debido a que las restricciones que exigen mejorar las distancias de sonicidad ocupan un lugar tan alto en la jerarquía, podrían empezar a modificarse también otros segmentos y contactos silábicos, que, sin embargo, aunque presentan malas distancias intersilábicas, prefieren ser fieles y se mantienen. Éste sería el caso de palabras con fricativas en posición implosiva seguidas de oclusivas (por ejemplo: asta, tostada), casos bastante frecuentes en español. Estos casos exhiben unas distancias intersilábicas muy malas, puesto que apenas hay distancia intersilábica:

\section{(15) $[\mathrm{F}, \mathrm{O}]=$ distancia intersilábica $=1 \otimes$}

Sin embargo, a pesar de ello, no se modifican. Esto tendría una explicación lógica, ya que hemos constatado que los segmentos sólo cambian dentro de la clase [+sonante] [-sonante] y, por lo tanto, habría una restricción en lo alto de la jerarquía que obligaría a conservar los rasgos [ \pm sonante]: $\mathrm{ID}_{[ \pm \text {sonante] }}$. Así, también se podría explicar por qué palabras como [át.las] o [at.mós.fe.ra] no suelen convertir las oclusivas implosivas en sonantes, a pesar de que así se mejoraría el perfil de sonicidad (las realizaciones de estas palabras pueden ser [ád.las], [á日.las], [át.las]; [að.mós.fe.ra], [a0.mós.fe.ra], [at.mós.fe.ra]). Por el contrario, si las variedades castellanomanchegas sí convirtieran estas oclusivas en sonantes, la restricción que estaría en lo alto de la jerarquía sería solo $\operatorname{ID}_{[++\operatorname{sonante}]}$ lo que permitiría que se dieran pronunciaciones con asimilaciones del tipo [ál.las], [am.mós.fe.ra]. En las respuestas del cuestionario de fonética del ALeCMan no hemos encontrado suficientes preguntas con oclusivas implosivas seguidas de segmentos sonantes ${ }^{21}$ como para realizar alguna generalización

21 Las palabras con oclusivas en posición implosiva suelen ser cultas, de ahí que no ocupen un lugar demasiado importante en el cuestionario del ALeCMan, centrado en los hablantes rurales y de la tercera edad. 
acerca de este comportamiento. Habrá que reunir más palabras de este tipo y ver cómo se comportan. Si tienden a mantener los segmentos [-sonante] como tales (es decir, si pronuncian atlas como [ád.las], [á日.las], [át.las]), la jerarquía para las hablas castellanomanchegas sería la siguiente:

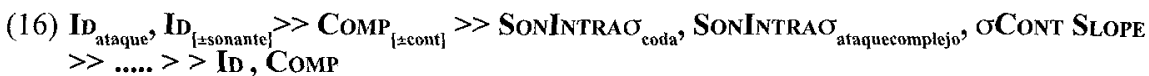

$\mathrm{Si}$, por el contrario, estas hablas presentan asimilaciones del tipo [ál.las], [am.mós. fe.ra], la restricción que se encontraría en un lugar importante de la jerarquía sería sólo ID [+sonante : $^{-}$

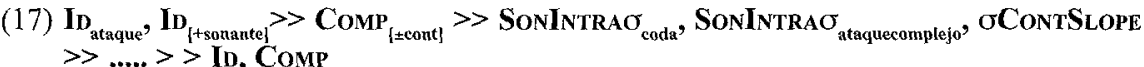

Según (16) y (17), dado que las nasales forman parte del grupo de las consonantes sonantes (Lloret 1997) también deberían modificar sus propiedades en contacto intersilábico con una oclusiva [N.O] o una fricativa [N.F]; sin embargo, se mantienen intactas, lo que hace pensar que también la restricción $\mathrm{ID}_{[t n a s a l]}$ ocupa un lugar importante en esta jerarquía, por encima de las restricciones relacionadas con las distancias de sonicidad.

Por último, existen otros contactos intersilábicos que, aunque no son los mejores, se conservan también intactos en estas y otras hablas. Por ejemplo, los contactos del tipo [vocal. laterall no se modifican - la lateral no se convierte en la rótica percusiva-, a pesar de que de esta manera la distancia intersilábica entre la vocal y la líquida se mejoraría. Obsérvese el siguiente ejemplo:

$$
\begin{array}{ll}
\text { [sá.la] } & {[\text { V.L] }=8-6=2} \\
\text { [sá.fa] } & {[\text { V.R] }=8-5=3}
\end{array}
$$

Aunque sala presenta una distancia intersilábica peor que la de sara, no se da el cambio sala > sara. Pero este hecho se explica fácilmente por la existencia de la ya mencionada restricción de fidelidad IDENTIDAD referente al ataque $\operatorname{ID}_{\text {ataque }}$ (los segmentos en correspondencia I-O deben conservarse idénticos si aparecen en posición de ataque).

Gracias a la jerarquía propuesta en (13), con sus matizaciones en (16) y (17), se explican las principales caracteristicas de la confusión en Castilla-La Mancha. Con todo, ya anunciamos que las generalizaciones aqui explicadas ( $r$ final de palabra $>$ l; l.oclusiva $>$ r.oclusiva) constituyen tan solo tendencias. Poquísimas veces un mismo contexto presenta confusión el $100 \%$ de las ocasiones. A esto hay que añadir que ni siquiera en un mismo hablante estas tendencias son "rotundas": aunque en ciertos contextos un hablante confunda en mayor medida las líquidas, ello no implica que siempre tenga que presentar confusión en tales contextos. La TO ha intentado explicar este hecho, este alto grado de variabilidad interna ${ }^{22}$. Para ello, ha habido trabajos que han propuesto que algunas restricciones son "flotantes" y ello es lo que motivaría que en unas ocasiones las líquidas se confundieran y en otras no.

22 En Jiménez y Lloret (2004) encontramos bibliografía sobre el tema. 
También ha habido otras propuestas que proponen que ciertas restricciones se encuentran sin ordenar, de modo que en caso de conflicto, varias soluciones quedan abiertas. Estas propuestas explicativas nos hacen pensar en la existencia de una jerarquía con las restricciones referentes a la confusión sin ordenar, o en la existencia de dos jerarquías simultáneas en un mismo hablante. De este modo, podríamos defender que el hablante castellanomanchego posee dos jerarquías: la jerarquía confundidora que es la que se ha propuesto aquí y hace que el hablante confunda $r$ y $l$ y la jerarquía de la norma estándar y escrita, que haría que el hablante distinguiera la $r$ y la $l$, al aparecer las restricciones de fidelidad en un lugar dominante en la jerarquía. Parece evidente que la primera jerarquía (la confundidora) estaría presente en situaciones informales, coloquiales... y la segunda (la de la norma) cobraría un papel más importante en situaciones de mayor formalidad. Con todo, el hecho de admitir la existencia de dos jerarquías debilitaría en cierto modo el poder explicativo de la TO, ya que su ventaja es precisamente ésta: a partir de una única jerarquía derivar todas las formas posibles en una lengua. Pero ya sabemos que las lenguas no son entes fijos y estables, sino que varían constantemente y el plano fonético es, justamente, el más sometido a variación, con lo que no sería tan descabellado plantear la existencia de más de una jerarquía. De cualquier modo, este es uno de los principales puntos de nuestro análisis que tendrá que mejorarse en un futuro, a medida que la teoría vaya avanzando.

\subsection{La variación lingüística en TO: análisis de la confusión en otras variedades}

La confusión de líquidas es un rasgo que se encuentra extendido por la geografía hispanohablante, adoptando realizaciones muy diversas en cada área. Desde el punto de vista de la optimidad, la multiplicidad de soluciones de la confusión se explica por la manera específica en que cada variedad ordena las restricciones lingüísticas universales, es decir, por el modo en que cada habla resuelve el conflicto entre las restricciones de fidelidad y de marcadez. Para ilustrar este hecho mediante unos datos concretos y, al mismo tiempo, comprobar, la validez de las restricciones propuestas en (4.1), a continuación exponemos la jerarquía de las restricciones de otras dos variedades confundidoras: (i) las que tienden a confundir las líquidas implosivas en beneficio de la lateral (con soluciones como amol por amor o puelto por puerto), característica de algunos puntos de Cuba, de Puerto Rico y de la República Dominicana (Aleza y Enguita 2002) y (ii) las que confunden también en posición de ataque, como por ejemplo ocurre en el catalán hablado en Alguer, el alguerés, con soluciones como prat por plat (plato) y crau por clau (clavo) (Veny 1982).

En la variedad lateralizante (i), las restricciones de marcadez referentes a las distancias de sonicidad ocuparán un lugar muy importante en su gramática, incluso por encima de otras restricciones de marcadez, como las que obligan a que los segmentos adyacentes compartan algún rasgo determinado y dan cuenta de los procesos de asimilación de rasgos. Así, la restricción COMPARTIR $[ \pm$ contimuo $]$ - tan importante en las variedades castellanomanchegas - en los puntos confundidores de Cuba, de Puerto Rico y de la República Dominicana, queda dominada por las de marcadez de sonicidad, lo que explica que sea tan frecuente el cambio $r$ $>1$ en coda silábica (tanto en interior como en final de palabra) ya que de este modo se consiguen las mejores distancias de sonicidad. La jerarquía de esta variedad sería la siguiente:

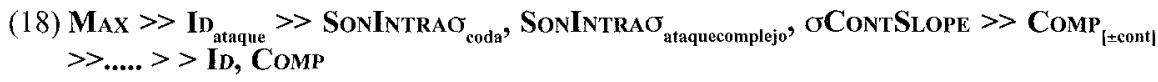


Como se desprende de (18), en estas hablas siguen siendo relevantes algunas restricciones de fidelidad puesto que, aunque tiendan a emerger las sílabas con las mejores distancias de sonicidad, lo que hace que se den cambios del tipo $r>1$, tales cambios no afectan nunca a la posición de ataque (IDENTIDAD ataque los protege) ni ocasionan el borrado de material fónico (MAXIMIDAD lo impide). Para comprobar e ilustrar que la jerarquía de (18) funciona y selecciona al candidato óptimo en las hablas lateralizantes de Hispanoamérica, seguidamente ejemplificamos la evaluación de diversas formas (puerto, comer, plato). En las tablas de evaluación de /puérto/ y /komér/ sólo copiamos las restricciones relevantes para el análisis (es decir, prescindimos de SONINTRA $\sigma_{\text {ataquecomplejo }}$ ya que los inputs evaluados no presentan ataques complejos).

(19) Evaluación del candidato óptimo para /puérto/:

\begin{tabular}{|c|c|c|c|c|c|}
\hline /puécto/ & In ataque & SONINTRAO $\sigma_{\text {cada }}$ & OCONTSLOPE & $\operatorname{CoMP}_{[ \pm \operatorname{cont}]}$ & In \\
\hline a.puér.to & & * & $* !$ & & \\
\hline b. puél.to & & & & $*$ & $*$ \\
\hline c. pués.to & & * & $*$ ! & & $*$ \\
\hline d. puén.to & & $*$ & $* !$ & & $*$ \\
\hline
\end{tabular}

- Distancias intrasilábicas ${ }_{\text {coda }}$ :

Candidato a: $[\mathrm{VR}]=3$

Candidato $b:[\mathrm{VL}]=2$;

Candidato $\mathrm{c}:[\mathrm{VF}]=6$ :

Candidato $\mathrm{d}:[\mathrm{VN}]=5$
Distancias intersilábicas:

Candidato a: $[$ R.O $]=4$

Candidato b: $[$ L.O $]=5$

Candidato c: $[\mathrm{F} . \mathrm{O}]=1$

Candidato $\mathrm{d}:[\mathrm{N} . \mathrm{O}]=2$
().

8

(20) Evaluación del candidato óptimo para /koméc/

\begin{tabular}{|c|c|c|c|c|c|}
\hline /komér/ & $\mathbf{I}_{\text {ataque }}$ & SONINTRAO $\sigma_{\text {coda }}$ & o ContSlope & $\operatorname{CoMP}_{[ \pm \operatorname{con} t]}$ & ID \\
\hline a. ko.mér & & $* !$ & & $*$ & \\
\hline b. ko.mél & & & & $*$ & * \\
\hline
\end{tabular}

- Distancias intrasilábicas ada

Candidato $1:[\mathrm{VR}]=3$

Candidato $2:[\mathrm{VL}]=2$

(21) Evaluación del candidato óptimo para /pláto/: (ahora sí usamos la jerarquía (18) completa, añadiendo la restricción de fidelidad que penaliza la supresión de elementos (MAXIMIDAD) y la que hace referencia a las secuencias con ataque complejo (SONINTRAO ataquecomplejo $_{\text {i }}$ ):

\begin{tabular}{|c|c|c|c|c|c|c|}
\hline /pláto/ & Max & $\mathrm{ID}_{\text {gatane }}$ & SONINIRAO ${ }_{\text {atanaecompleio }}$ & SONINTRAO & $\mathrm{COMP}_{1+\text { conti }}$ & ID \\
\hline a.plá to & & & * & Pिमे? & 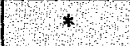 & \\
\hline b.prá to & & $* !$ & & 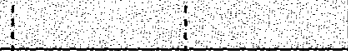 & $*$ & $*$ \\
\hline c.pá. to & $* !$ & & 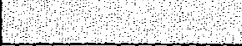 & 1 & $*$ & \\
\hline
\end{tabular}


Mediante estas tablas queda comprobada, por tanto, la jerarquía de restricciones propuesta para las hablas lateralizantes de América: la restricción de fidelidad posicional $\mathrm{ID}_{\text {ataque }}$ y la que penaliza la elisión de segmentos MAx son las que ocupan un lugar más importante dentro de la ordenación, dominando al resto de restricciones. Aunque en esta variedad las líquidas se modifican en posición de coda con el propósito de mejorar las distancias de sonicidad intrasilábicas e intersilábicas, los ataques complejos ( $\mathrm{pr}, \mathrm{br}, \mathrm{pl}$, etc.) siempre se mantienen "intactos"; de ahí que la restricción de fidelidad $\mathrm{ID}_{\text {ataque }}$ esté en un lugar tan alto en la jerarquía. Siguiendo en el orden a estas restricciones nos encontramos con las restricciones de marcadez referentes a los perfiles de sonicidad: SonInTRa $\sigma_{\text {coda }}$, $\sigma$ CONTSLOPE, SonINTRA $\sigma_{\text {ataquecomplejo }}$. Éstas, a la vista de los datos analizados, no se encuentran ordenadas entre sí, sino que tienen la misma importancia relativa dentro de la jerarquía. Y ya hemos visto que las tres restricciones favorecen la emergencia de la lateral en posición implosiva. Finalmente, en el lugar más bajo de la jerarquía, encontramos una restricción de fidelidad más general ID que pugna por que los segmentos del output se mantengan fieles a los del input. Esta especial jerarquía de las restricciones justifica por qué en algunas variedades del español de América las vibrantes implosivas, tanto a final de palabra como en interior de palabra, emergen como laterales (Puelto Rico, mi amol), pero, cuando aparecen como segundo segmento de un ataque complejo, mantienen todas sus propiedades.

Por su parte, para las variedades como el catalán de Alguer, que también modifican las características de la lateral de un ataque complejo, convirtiéndola en rótica, la jerarquía de las restricciones universales tendrá que ser la postulada en (22):

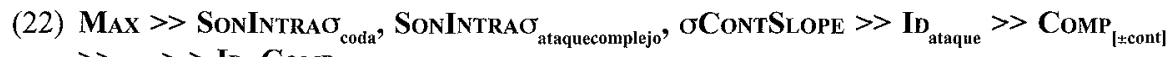
$>$..... $>>$ ID, COMP

En el alguerés lo más importante es respetar las restricciones referentes a la sonicidad, de ahí que ocupen un lugar tan importante en la jerarquía. En esta variedad del catalán, la confusión de líquidas afecta también al segundo elemento de un ataque complejo. En estos casos, la $l$ del ataque complejo pasa a realizarse como una $r$, ya que así se consigue una sílaba con una distribución de la sonicidad más homogénea, como postulaba Clements (1990: 304). Por ello, la restricción de fidelidad referente a las propiedades del ataque $\left(\mathrm{ID}_{\text {ataque }}\right)$ pasa a ocupar en la jerarquía de esta variedad del catalán un lugar menos dominante, por debajo de todas las referentes a la sonicidad. La tabla (23) evalúa el candidato óptimo según la jerarquía establecida en (22) para el input /plát/ (plato):

(23)

\begin{tabular}{|c|c|c|c|c|c|c|}
\hline /plát/ & Max & SONINTRa $\sigma_{\text {coda }}$ & SONINTRAO $\sigma_{\text {ataquecomplejo }}$ & бCONTSLOPE & $\operatorname{CoMP}_{[ \pm \text {tont }]}$ & $\mathbf{I D}_{\text {ataque }}$ \\
\hline a. plát & & * & *! & 1 & & \\
\hline b. prát & & * & & i & & + \\
\hline c. pát & $* !$ & * & 8 & 1 & & \\
\hline
\end{tabular}

- Distancias intrasilábicas entre el ataque complejo y el núcleo:

Candidato a: $[\mathrm{ORV}]=3$

Candidato $\mathrm{b}:[\mathrm{OLV}]=2$-) Presenta una distribución de la sonicidad más homogénea Candidato c: [OV] (no hay ataque complejo) 
En todos los candidatos analizados la semisílaba final [-at] es mala porque presenta una oclusiva en la coda (Clements 1990: 305). Sin embargo, otras restricciones específicas de identidad, como explicábamos en (4.1) evitan el paso de la oclusiva a consonantes menos sonantes $^{23}$ y MAX, ordenada por arriba, evita su elisión.

\section{Conclusiones}

Tras revisar los materiales del ALeCMan, se ha constatado que la confusión de líquidas es un rasgo característico de algunos puntos de Toledo, Ciudad Real y Albacete (Imagen I). Allí, aunque el fenómeno confundidor es bastante asistemático, hay dos contextos en que se da con mayor frecuencia: (a) a final de palabra, la -r pasa a -l (Pilal por Pilar, bebel por beber, etc.) y (b) en interior de palabra, seguida de oclusiva, la -1 se convierte en $-\mathrm{r}$ (arba por alba, arto por alto, etc.). Valiéndonos del marco teórico de la Teoría de la Optimidad, hemos intentado esclarecer por qué estos dos contextos desencadenan la confusión de líquidas. Si bien el análisis propuesto (apartado 4) no es perfecto y presenta algunas imprecisiones -todo lo relativo al valor del rasgo [ $t$ continuo] de las líquidas- supone un avance frente a las anteriores explicaciones articulatorias y permite dar cuenta de la confusión en otras variedades del español. Así, la TO ha puesto de manifiesto los principales detonantes de la confusión y ha explicado la multiplicidad de soluciones en las distintas variedades confundidoras: dependiendo de cómo resuelva cada variedad el conflicto entre las restricciones de marcadez y de fidelidad, es decir, según qué jerarquía presenten las restricciones universales en cada habla, se dará un tipo de confusión u otra. Así, sabemos que en algunas variedades del español la $r$ y la $l$ se confunden para mejorar las distancias de sonicidad intersilábicas e intrasilábicas; es decir, para respetar las exigencias de restricciones del tipo SonINTRao ${ }_{\text {coda }}$, oConTSlope y SONINTRAG $\sigma_{\text {ataquecomplejo }}$. En ese sentido, ahora se entiende por qué unas veces emerge la lateral (para respetar SONINTRAO $\sigma_{\text {coda }}, \sigma$ CONTSLOPE) y otras la rótica (para respetar SONINTRA $\sigma_{\text {ataquecompleio }}$ ). Lo que antes resultaba tan "caótico", visto desde la TO no lo es tanto. Pero éstas no son las únicas restricciones de marcadez que pueden entrar en conflicto con las de fidelidad, sino que también hay ocasiones en que la $r$ y la $l$ se confunden debido al requisito de similitud que existe entre ciertos segmentos adyacentes: especialmente, los segmentos en posición de coda que, debido a su naturaleza débil, tienden a adoptar rasgos de los segmentos que las siguen; es decir, la $r$ y la $l$ implosivas también pueden confundirse para satisfacer la restricción $\operatorname{Comp}_{\text {Itcont }]}$ (en ese sentido, en las hablas confundidoras de Castilla-La Mancha, siempre que una de las dos líquidas vaya seguida de una oclusiva, la solución de la confusión es la rótica, pues ésta es la líquida que comparte el rasgo [-continuo] con los segmentos oclusivos.

En definitiva, este trabajo constituye una propuesta de análisis de la confusión en términos de optimidad, pero tendrá que verse mejorado en futuras investigaciones. Concretamente, tendrá que matizarse, por un lado, todo lo relativo a la variación libre de la confusión - habrá que determinar por qué un mismo contexto desencadena confusión en algunas ocasiones, pero en otras no. Y, por otro lado, habrá que fundamentar mejor la caracterización de la lateral como [+continuo] y la rótica y la oclusiva como [-continuol y su relación con los procesos de espirantización.

23 Este hecho se da, por ejemplo, en el español de Chile, donde las oclusivas implosivas se convierten en semivocales (Martinez Gil, 1997). 


\section{Referencias bibliográficas}

Alarcos Llorach, E. (1965): Fonología española, Madrid, Gredos. Se ha empleado la edición de 1991.

Aleza, M. y Enguita, J. Mª (2002): El español de América: aproximación sincrónica, Valencia, Tirant lo Blanch.

Alonso, A. y Lida, R. (1945): "Geografia fonética: -I y $-r$ implosivas en español", Revista de Filología Hispánica, vII, 313-345. También en Alonso, A. y Lida, R. (1961): “'R' y 'L' en España y América". En Estudios lingïísticos. Temas hispanoamericanos, Madrid, Gredos, 213-267. En el trabajo se cita por esta última.

Bat-El, O. (1996): "Selecting the best of the worst: the grammar of Hebrew blends", Phonology, XIII, 283-328.

Beckman, J. N. (1999): "Positional faithfulness", tesis doctoral de 1998, University of Masschusetts, Amherst, New York, Gorland.

Bonet, E. y Lloret, M-R. (1998): Fonologia catalana, Ariel, Barcelona.

Catalán, D. (1989): "Entorno a la estructura silábica del español de ayer y del español de mañana". En El español, origenes de su diversidad, Madrid, Paraninfo, 77-104.

Clements, G. N. (1990): "The role of the sonority cycle in core syllabification". En Kingston, J. y Beckman, M. E. (eds.): Papers in laboratory phonology I: between the grammar and physics of speech, Cambridge, Cambridge University Press, 283-333.

Colina, S. (2007): "Optimality-theoretic advances in our understanding of Spanish syllable structure". En Martínez-Gil, F. \& Colina, S. (eds.): Optimality-Theoretic Studies in Spanish, Amsterdam/ Philadelphia, John Benjamins, 172-204.

Côté, M-H. (2000): Consonant Cluster Phonotactics: A perceptual Approach, tesis doctoral, MIT. Disponible en red: http://www.roa.rutgers/, $\mathrm{n}^{\circ} 548$.

Chomsky, N. y Halle, M. (1968): The Sound Pattern of English, New York, Harper and Row.

García Jurado, M. A., Guirao, M. y Rosso, E. (1991): "La influencia de la duración en la identificación de las líquidas". En Actas del III Congreso Internacional del Español de América, 393-403.

García Mouton, P. y Moreno Fernández, F. (1994): "El Atlas Lingüístico y etnográfico de Castilla-La Mancha. Materiales fonéticos de Ciudad Real y Toledo". En García Mouton (ed.): Geolingüística. Trabajos europeos, Madrid, CSIC, 111-154.

García Mouton, P. y Moreno Fernández, F. (dir.): Atlas Lingüistico y etnográfico de Castilla-La Mancha. Los materiales comenzaron a editarse por internet en mayo 2003; pueden consultarse en: http://www.uah.es/otrosweb/alecman/

Grammont, M. (1971): Traité de phonétique, Paris, Delagrave.

González Salgado, J. A. (2003): "La fonética en las hablas extremeñas", Revista de Estudios Extremeños, lix, 2, 589-619.

Jiménez, J. (1999): L'estructura sil làbica del català, Biblioteca Sanchis Guarner, València / Barcelona, Institut Interuniversitari de Filologia Valenciana, Publicacions de l'Abadia de Montserrat.

Jiménez, J. y Lloret, M-R (2004): "Darreres tendències en fonologia generativa", Estudios Catalanes. Revista Internacional de lengua, literatura y cultura catalanas, año 2, número 2, Santa Fe, República Argentina, Universidad Nacional del Litoral, 111-129. Disponible en red: http://www. uv.es/foncat, $\mathrm{n}^{\circ} 2$.

Kirchner, R. (1998): An efford-based approach to consonant lenition, tesis doctoral, UCLA. Disponible en red: http://Www.roa.rutgers, $\mathrm{n}^{\circ} 30$.

Llorente Maldonado de Guevara, A. (1962): "Fonética y fonología andaluza", Revista de Filología Española, 45, 227-241.

Llorente Maldonado de Guevara, A. (1965): "Algunas características lingüísticas de La Rioja en el marco de las hablas del valle del Ebro y de las comarcas vecinas de Castilla y Vasconia", Revista de Filología Española, 48, 321-330. 
Lloret, M-R. (1997): "Sonorant dissimilation in the Iberian languages", Martínez Gil, F. \& Morales Front, A.: Issues in the phonology and morphology of the Major Iberian Languages, Georgetown University Press, 127-150.

Lloret, M.R. y Jiménez, J. (2007): "Prominence driven epenthesis: evidence from Catalan", ms., Universitat de Barcelona / Universitat de València. Disponible en red: http://www.uv.es/foncat, $\mathrm{n}^{\circ} 19$.

Lombardi, L. (1999): "Positional Faithfulness and voicing assimilation in Optimality Theory", Natural Language and Linguistic Theory, 17, 267-302.

Malmberg, B., (1965): “La structure syllabique de l'espagnol". En Estudio de Fonética Hispánica, Madrid, CSIC, 3-28.

Martínez Gil, F. (1997): "Consonant vocalization in Chilean Spanish: A serial vs. a constraint-based approach", Probus, 9, 167-202.

Mascaró, J. (1991): "Iberian spirantization and continuant spreading", Catalan Working papers in Linguistics 1991, Bellaterra, Universitat Autònoma de Barcelona, 167-179.

Murray, R. W. y Vennemann, T. (1983): "Sound change and syllable structure in Germanic phonology", Language, 59, 514-528.

Navarro Tomás, T. (1975): “Áreas geográficas de consonantes finales”, Capitulos de Geografía Lingüistica de la Península Ibérica, Bogotá, Publicaciones del Instituto Caro y Cuervo, XXXV, 187-191.

Palmada, B. (1997): "Continuant spreading and feature organization", Martínez Gil, F. \& Morales Front, A. (eds.): Issues in the phonology and morphology of the Major Iberian Languages, Georgetown University Press, 299-313.

Parker, S. (2002): Quantifying the sonority hierarchy, tesis doctoral, Graduate Linguistic Association, University of Massachusetts, Amherst.

Piñeros, C-E. (2001): "Non-inherent voicing and ways to fix it", ms., University of Iowa. Disponible en red: http://www.roa.rutgers, $n^{\circ} 483$.

Prince, A. y Smolensky, P. (1993): Optimality Theory. Constraint interaction in Generative Grammar, manuscrito de 1993. Aquí se ha empleado la versión del 2002, disponible en red: http://www.roa rutgers.edu/

Quilis-Sanz, M. J. (1998): "Las consonantes [-r] y [-1] implosivas en Andalucía", Revista de Filología Española, 125-156.

Veny, J. (1982): Els parlars catalanas: sintesi de dialectologia, Palma de Mallorca, Moll.

Wheeler, M. W. (2005): The phonology of Catalan, Oxford, Oxford University Press.

Wright, R. (2004): "A review of perceptual cues and cue robustness", Hayes, B., Kirchner, R. y Steriade, D. (eds.): Phonetically based phonology, Cambridge, Cambridge University Press, 34-57. 